\title{
Progress in timing Resistive Plate Chambers
}

\author{
A. Blanco ${ }^{\mathrm{a}}$, N. Carolino ${ }^{\mathrm{a}}$, C.M.B.A. Correia ${ }^{\mathrm{a}, \mathrm{b}}$, R. Ferreira-Marques ${ }^{\mathrm{a}, \mathrm{c}}$, \\ P. Fonte ${ }^{\mathrm{a}, \mathrm{d}, *}$, A. Gobbi ${ }^{\mathrm{e}}$, D. González-Díaz ${ }^{\mathrm{f}}$, M.I. Lopes ${ }^{\mathrm{a}, \mathrm{c}}$, L. Lopes ${ }^{\mathrm{a}}$, \\ M.P. Macedo ${ }^{\mathrm{b}, \mathrm{d}}$, A. Mangiarotti ${ }^{\mathrm{g}}$, V. Peskov ${ }^{\mathrm{h}}$, A. Policarpo ${ }^{\mathrm{a}, \mathrm{c}}$ \\ ${ }^{a}$ Laboratório de Instrumentação e Física Experimental de Particulas (LIP), Universidade de Coimbra, Dep. de Fisica, \\ 3004-516 Coimbra, Portugal \\ ${ }^{\mathrm{b}}$ Centro de Electrónica e Instrumentação, Departamento de Física, Universidade de Coimbra, 3004-516 Coimbra, Portugal \\ ${ }^{\mathrm{c}}$ Departamento de Física, Universidade de Coimbra, 3004-516 Coimbra, Portugal \\ ${ }^{\mathrm{d}}$ Instituto Superior de Engenharia de Coimbra, Rua Pedro Nunes, 3030-199 Coimbra, Portugal \\ ${ }^{\mathrm{e}}$ Gesellschaft für Schwerionenforschung (GSI), Planckstr. 1, 64291 Darmstadt, Germany \\ ${ }^{\mathrm{f}}$ GENP, Departamento de Física de Partículas, University Santiago de Compostela, 15782 Santiago de Compostela, Spain \\ ${ }^{\mathrm{g}}$ Physikalisches Institut der Universität Heidelberg, Philosophenweg 12, D-69120 Heidelberg, Germany \\ ${ }^{\mathrm{h}}$ Physics Department, Royal Institute of Technology, Stockholm Physics Center, Stockholm 10691, Sweden
}

Available online 12 August 2004

\begin{abstract}
Timing RPCs are Resistive Plate Chambers made with glass and metal electrodes separated by precision spacers. Typical gas gaps are a few hundred micrometers wide. Such counters were introduced in 1999 and have since reached timing accuracies below $50 \mathrm{ps} \sigma$ with efficiencies above $99 \%$ for MIPs. Applications in high-energy physics have already taken place with several more under study.

Some recent developments include the extension of the counting rate capability by over one order of magnitude, to $25 \mathrm{kHz} / \mathrm{cm}^{2}$, with time resolutions below $100 \mathrm{ps} \sigma$. A prototype RPC-based Positron Emission Tomograph yielded a reconstructed point-source resolution of $0.6 \mathrm{~mm}$ FWHM and a modified timing RPC design, featuring $50 \mu \mathrm{m}$ pitch anode strips, allowed to reach extremely good position resolution for hard X-rays in digital readout mode.

An analytically solvable model has allowed us to clarify the basic factors influencing the time resolution. (C) 2004 Elsevier B.V. All rights reserved.
\end{abstract}

PACS: $29.40 . \mathrm{Cs}$

Keywords: RPCs; ToF

\footnotetext{
*Corresponding author. LIP, Dept. de Física da Universidade de Coimbra P-3004-516, Portugal. Tel.: + 351-239-833465; fax: $+351-239-822358$.

E-mail address: fonte@lipc.fis.uc.pt (P. Fonte).
} 


\section{Introduction}

Resistive Plate Chambers (RPCs) with timing resolutions below 100 ps $\sigma$ for minimum ionizing particles have been recently developed [1,2]. This type of detector, operating at atmospheric pressure with non-flammable gases, seems well suited for high-granularity time-of-flight (ToF) systems, providing performances comparable to the scintillator-based ToF technology but offering a significantly lower price per channel, compact mechanics and magnetic field compatibility.

In this study we describe some recent advances of timing RPC technology with emphasis on the physical understanding of the device based on an analytical treatment. Although such an approach cannot reproduce all operational details, considerable insight is gained on the fundamental factors influencing the RPC performance.

\section{Very high rate and position resolution}

Modified thin-gap RPCs made of low resistivity materials, for example Si or GaAs, combine a high counting rate capability (up to $10^{5} \mathrm{~Hz} / \mathrm{mm}^{2}$ at gains up to $10^{5}$ ), approaching that of metallic PPACs, with a still efficient protection of the electronics from sparks. An excellent position resolution $(\approx 50 \mu \mathrm{m})$ for high-energy $\mathrm{X}$-rays was obtained in a simple counting (digital) mode without any analog interpolation $[3,4]$.

Similar counters, but with a sensitivity to UV and visible photons, have also been introduced $[4,5]$.

\section{RPC-based Positron Emission Tomography}

Time resolutions of $300 \mathrm{ps}$ FWHM have been measured for $511 \mathrm{keV}$ photon pairs (equivalent to 90 ps $\sigma$ for each photon), with possible application to whole-body human PET imaging [6]. Recently, a reconstructed position resolution of $0.6 \mathrm{~mm}$ FWHM, without any use of enhancement techniques, has been demonstrated [7], rendering the sub-millimetric imaging of small animals also an attractive perspective.

\section{High-rate timing RPCs}

The extension of the counting rate capabilities of Resistive Plate Chambers (RPC) has been often an important part of the requirements for new applications. The maximum counting rate capability of timing RPCs was increased from almost $2 \mathrm{kHz} / \mathrm{cm}^{2}$ [8] to $27 \mathrm{kHz} / \mathrm{cm}^{2}$ by using a low resistivity plastic anode [9]. A time resolution better than $100 \mathrm{ps} \sigma$ for $\gamma$-photons, essentially independent of the counting rate, was observed.

\section{Analytical model of RPC timing properties}

It is always interesting to understand the intrinsic limitations of a particular detection concept arising from the physical processes taking place in the device: they set the ultimate goal for its performances. In RPCs, given the planar geometry, the counter generated current signal is exponentially growing up to the level where the space charge effect is present. It is then important to realize that, in the same time interval, the voltage signal at the output of the pre-amplifier will also grow exponentially, independently of any detail of the transfer function [10,11]. Assuming an ideal response of the timing discriminator, the overall behavior of the electronic chain can be described with a direct threshold on the total flowing current or equivalently on the total multiplication [11]. Then the intrinsic timing properties of an RPC are determined by the fluctuations in the current growth, i.e. in the number of clusters and in the multiplication. The dynamics of the avalanche development has been studied in proportional counters for non-electronegative [12] as well as for electronegative mixtures [13]. Based on the knowledge thus accumulated, the intrinsic time response function of an RPC $\rho_{T}$ was initially calculated for a single avalanche [14] and afterwards extended to the general situation of several avalanches growing simultaneously with their number fluctuating according to a Poisson distribution [11]. The case of an electronegative mixture was considered explicitly in Ref. [15] 
arriving at the formula

$$
\begin{aligned}
\rho_{T}(\tau)= & \frac{n_{0}^{\prime}}{\mathrm{e}^{n_{0}^{\prime}}-1} \frac{\mathrm{e}^{\left(\tau_{\text {th }}-\tau\right)-\exp \left(\tau_{\mathrm{th}}-\tau\right)}}{\sqrt{n_{0}^{\prime} \mathrm{e}^{\left(\tau_{\mathrm{th}}-\tau\right)}}} \\
& \times I_{1}\left(2 \sqrt{n_{0}^{\prime} \mathrm{e}^{\left(\tau_{\mathrm{th}}-\tau\right)}}\right)
\end{aligned}
$$

where $I_{n}$ denotes the modified Bessel Function of integer order $n, \tau_{\text {th }}=\ln \left(r m_{\mathrm{th}}\right)$ is an overall time offset determined by the timing threshold in multiplication $m_{\mathrm{th}}, \tau=t s$ is the non-dimensional time scale expressed in time units of $1 / s$ with $s=$ $\alpha^{\star} v_{\mathrm{d}}$ and $n_{0}^{\prime}=n_{0} r$ is the effective number of clusters $n_{0}$ in the active fraction of the gap [11] reduced by a factor $r$ due to electron attachment. The gas swarm parameters are the first Townsend coefficient $\alpha$, the electron attachment coefficient $\eta$ and the electron drift velocity $v_{\mathrm{d}}$. For simplicity, $\alpha^{\star}=(\alpha-\eta)$ and $r=1-\eta / \alpha$ are adopted. It has to be emphasized that Eq. (1) has been derived under the hypothesis that the space chargeinduced saturation is not present at the level of the threshold $m_{\text {th }}$ and that non-equilibrium phenomena in the avalanche dynamics are negligible at the field strengths of concern.

The fundamental features of Eq. (1) are the following three: (i) the threshold $m_{\text {th }}$ enters only in the time offset $\tau_{\text {th }}$ and does not influence the shape of the response; (ii) once the non-dimensional time scale $\tau$ is introduced, the effect of the particular mixture considered is factored out; and (iii) the shape of $\rho_{T}$ in the variable $\tau$ is uniquely controlled by $n_{0}^{\prime}$. As a consequence, in the new nondimensional quantity $\tau$, all the central moments of order $>1$ are a universal function of $n_{0}^{\prime}$ alone. In particular, series expansions have been developed for $\langle\tau\rangle$ and for the variance $\left(K\left(n_{0}^{\prime}\right)\right)^{2}[16]$. The corresponding rms deviation $K\left(n_{0}^{\prime}\right)$ is depicted in Fig. 1. The shape of Eq. (1) is asymmetric with a tail toward large delays that rapidly decreases for $n_{0}^{\prime}>3$ : for this reason it is difficult to estimate experimentally the rms deviation and usually a Gaussian fit is performed in an interval $\pm 1.5 \sigma$ wide to derive the resolution. If a similar procedure is applied to Eq. (1), a distortion of the universal curve is produced as shown also in Fig. 1. Since $n_{0}^{\prime}$ is directly connected to the intrinsic counter efficiency $\varepsilon$ by $n_{0}^{\prime}=-\ln (1-\varepsilon)$, a universal relation

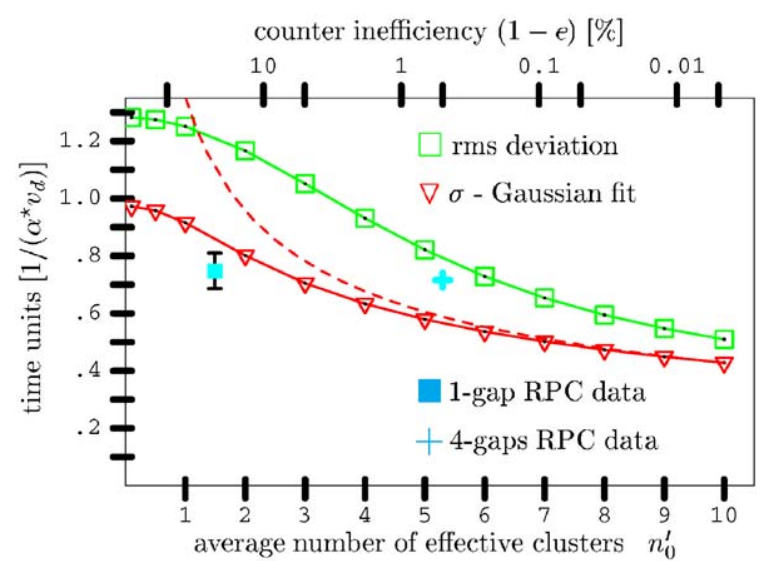

Fig. 1. Resolution, expressed in time units of $1 /\left(\alpha^{\star} v_{\mathrm{d}}\right)$, versus the average number of effective clusters $n_{0}^{\prime}$. The dashed line represents the asymptotic $1 / \sqrt{n_{0}^{\prime}}$ scaling.

between the resolution, expressed in units of $1 / s$, and $\varepsilon$ is finally obtained.

The measured timing accuracy is seen to depend on the applied voltage when, on the other hand, $\varepsilon$ is almost saturated [6], implying that the observed variation of the first is mostly due to the time scale $1 / s$ (see Fig. 4 of Ref. [11]). In fact, if the experimental resolution is re-expressed in terms of $1 / s$ (extracted with the electronic technique of Ref. [10]), it becomes constant and only the average is plotted in Fig. 1 (without correcting for the charge-time correlation, see Ref. [11]).

The formalism developed so far may be generalized to include the contribution of a random fluctuation in the electric field strength $E$ (and hence in the time scale $1 / s$ ): its physical origin stems from the mechanical tolerances of the gap width and from the local voltage drops caused by the charge produced in the preceding avalanches and not removed fast enough through the resistive electrode. Because the present purpose is mainly qualitative, it is of great advantage to consider the rms deviation, whose propagation properties are known. In particular, since the time delay is $\langle\tau\rangle / s_{0}$ (where $s_{0}=s$ at the average electric field strength $E_{0}$ ), neglecting all contributions of order next to leading, its final rms deviation is just the quadratic addition of the two relative ones corresponding to the intrinsic fluctuation in total multiplication 
$\left(K\left(n_{0}^{\prime}\right)\right)$ and to the alteration of the applied field $E$, yielding

$\operatorname{rms}_{T}=\frac{1}{s_{0}}\left[\left(K\left(n_{0}^{\prime}\right)\right)^{2}+\left(\frac{\langle\tau\rangle}{s_{0}}\left(\frac{\mathrm{d} s}{\mathrm{~d} E}\right)_{E=E_{0}} \mathrm{rms}_{\Delta E}\right)^{2}\right]^{1 / 2}$.

Eq. (2) expresses the fact that the perturbation of the field $E$ has a bigger influence on the overall timing resolution if the variation of $s$ with $E$ is steeper or the average delay $\langle\tau\rangle / s_{0}$ is longer. The two terms inside the square root of Eq. (2) together with their final quadratic sum are depicted in Fig. 2 for the case of a 1-gap $(0.3 \mathrm{~mm}$ wide) RPC operated in the standard mixture [6] at the maximum practical bias voltage of $3.2 \mathrm{kV}$. The value of $s_{0}=1.1 \times 10^{-2} \mathrm{ps}^{-1}$ as well as of the slope $\mathrm{d} s / \mathrm{d} E=2.0 \times 10^{-4} \mathrm{ps}^{-1} \mathrm{kV}^{-1} \mathrm{~cm}$ are taken again from measurements performed with the electronic technique of Ref. [10]. For an rms field perturbation of $10 \%$ the worsening of the resolution is $\approx 20 \%$. As an illustrative example, the cases of $m_{\mathrm{th}}=10^{6}$ and $m_{\mathrm{th}}=10^{4}$ are also compared to the more usual $m_{\mathrm{th}}=10^{5}$ [11]. If the origin of $\mathrm{rms}_{\Delta E} / E$ is just the gap-thickness non-uniformity, it is simply $\mathrm{rms}_{\Delta E} / E=\mathrm{rms}_{\Delta d} / d$ and the necessary mechanical tolerance to achieve a certain timing accuracy can be directly read off Fig. 2.

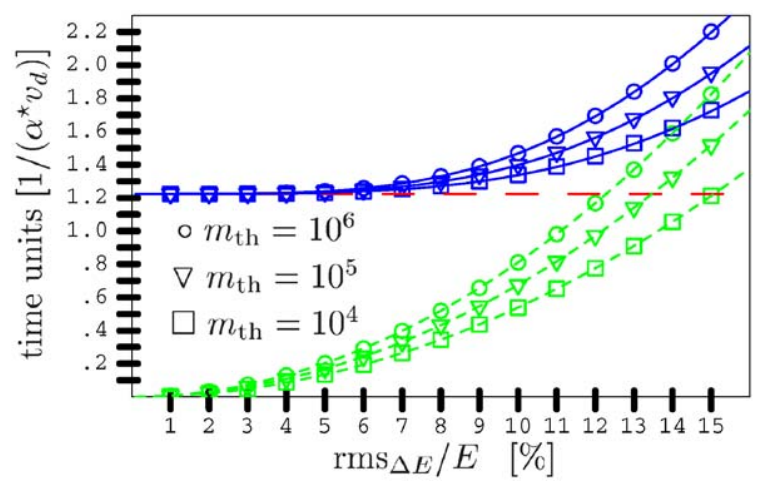

Fig. 2. Dependence on $\mathrm{rms}_{\Delta E} / E$ of the total timing resolution (rms continuous lines) and of each of the two contribution in Eq. (2) intrinsic (long dashed line) and due to the fluctuations in the field strength (small dashed lines). Three hypotheses are considered for $m_{\mathrm{th}}$.

\section{Conclusions}

Timing RPCs are an emergent particle detection technology that can provide very good time and position resolution at quite respectable counting rates. Applications to time-of-flight detectors for high-energy or nuclear physics to $\gamma$-, X-, or UV photon imaging have been already implemented or are under study. A few relevant examples have been described.

The fundamental timing properties of the device can be understood on the basis of an analytic model, yielding predictions for the dependence of the time resolution on factors like the applied voltage, primary ionization density, number of gas gaps, threshold level and tolerance to electric field inhomogeneities arising from mechanical distortions or rate effects.

\section{Acknowledgements}

This work was co-financed by Fundação para a Ciência e a Tecnologia project POCTI/FNU/ 49513/ 2002 and FEDER. One of the authors (AM) acknowledges support from the German BMBF under contract 06HD154.

\section{References}

[1] P. Fonte, A. Smirnitski, M.C.S. Williams, Nucl. Instr. and Meth. A 443 (2000) 201.

[2] P. Fonte, et al., Nucl. Instr. and Meth. A 449 (2000) 295.

[3] I. Crotty, et al., Nucl. Instr. and Meth. A 505 (2003) 203.

[4] C. Iacobaeus, et al., Nucl. Instr. and Meth. A 513 (2003) 244.

[5] T. Francke, V. Peskov, I. Rodionov, P. Fonte, High Rate (up to $\left.10^{5} \mathrm{~Hz} / \mathrm{cm}^{2}\right)$, High Position Resolution $(50 \mu \mathrm{m})$ Photosensitive RPCs, 7th RPC Workshop, 20-22 October 2003, Clermont-Ferrand, France, Nucl. Instr. and Meth. A, submitted.

[6] A. Blanco, et al., Nucl. Instr. and Meth. A 508 (2003) 88.

[7] A. Blanco, N. Carolino, C.M.B.A. Correia, et al., An RPC-PET prototype with high spatial resolution, 7th RPC Workshop, 20-22 October 2003, Clermont-Ferrand, France, Nucl. Instr. and Meth. A, submitted.

[8] A. Akindinov, et al., Nucl. Instr. and Meth. A 490 (2002) 58.

[9] L. Lopes, et al., Development of high-rate timing RPCs, 7th RPC Workshop, 20-22 October 2003, Clermont- 
Ferrand, France, Nucl. Instr. and Meth. in Phys. Res. A, submitted.

[10] A. Blanco, et al., IEEE Trans. Nucl. Sci. NS-48 (2001) 1249.

[11] A. Blanco, et al., Nucl. Instr. and Meth. A 513 (2003) 8.

[12] H. Genz, Nucl. Instr. and Meth. 112 (1973) 83.

[13] W. Legler, Z. Naturforschung 16a (1961) 253.
[14] A. Mangiarotti, et al., Nucl. Instr. and Meth. A 482 (2002) 192.

[15] A. Mangiarotti, P. Fonte, A. Gobbi, Exactly solvable model for the time response function of RPCs, 7th RPC Workshop, 20-22 October 2003, Clermont-Ferrand, France, Nucl. Instr. and Meth. A, submitted.

[16] A. Gobbi, et al., Nucl. Instr. and Meth. A 508 (2003) 23. 